\title{
COGROWTH SERIES OF FREE PRODUCTS OF FINITE AND FREE GROUPS
}

\author{
DMITRI KUKSOV \\ Department of Mathematics, Brigham Young University, 292 TMCB, Provo, Utah 84602, USA
}

(Received 12 February, 1997)

1. Introduction. Let $A=\left\{a_{1}, \ldots, a_{n}, a_{1}^{-1}, \ldots, a_{n}^{-1}\right\}$ and iteration of $A$ denoted by $A^{\star}$ to be the set of words in $A$ (including the empty word). Let $S \subseteq A^{\star}$; then the growth function of the set $S$ is the function $\Gamma(l)=$ number of words in $S$ of length $l$. For $m \leq n$ let $\vec{a}=\left(a_{i_{1}}, \ldots, a_{i_{m}}\right)$, where $i_{k} \in\{1, \ldots, n\}$ are different; then the relative growth function with respect to $\vec{a}$ is the function $\Gamma_{\vec{a}}\left(l, l_{1}, \ldots, l_{m}\right)=$ number of words in $S$ of length $l+l_{1}+\ldots+l_{m}$ having (for each $k$ ) $l_{k}$ total occurrences of $a_{i_{k}}$ and ${a_{i_{k}}}^{-1}$. Then, the growth series of $S$ and the relative growth series of $S$ with respect to $\vec{a}$ are the functions

$$
\gamma(S ; t)=\sum_{l=0}^{\infty} \Gamma(l) t^{l}
$$

and

$$
\gamma_{\vec{a}}\left(S ; t, t_{i_{1}}, \ldots, t_{i_{m}}\right)=\sum_{l, l_{1}, \ldots, l_{m}=0}^{\infty} \Gamma_{\vec{a}}\left(l, l_{1}, \ldots, l_{m}\right) t^{l} t_{i 1}^{l 1} \cdots t_{i m}^{l m},
$$

respectively. The set $S$ or variables $t, t_{i_{1}}, \ldots, t_{i_{m}}$ will be omitted when understood. Thus, we will sometimes write $\gamma_{\vec{a}}(S)$ or $\gamma_{\vec{a}}\left(t, t_{i_{1}}, \ldots, t_{i_{m}}\right)$ instead of $\gamma_{\vec{a}}\left(S ; t, t_{i_{1}}, \ldots, t_{i_{m}}\right)$. Clearly, $\gamma(t)=\gamma_{\vec{a}}(t, \ldots, t)$. Let

$$
1 \rightarrow N \rightarrow \mathbf{F}_{n} \stackrel{\phi}{\rightarrow} G \rightarrow 1
$$

be a presentation for the group $G$, where $\mathbf{F}_{n}=\left\langle a_{1}, \ldots, a_{n}\right\rangle$ is the free group. Also let $W(k)$ be the set of freely reduced words $w$ in $\mathbf{F}_{n}$ having length $k$ and representing the identity of $G$. The function $\Upsilon(k)=|W(k)|$ is called the cogrowth function for this presentation (it is also the growth function of $N$ ), and the function

$$
v(t)=\sum_{j=0}^{\infty} \Upsilon(j) t^{j}
$$

is called the cogrowth series of the presentation. Let

$$
\alpha(k)=\sum_{j=0}^{k} \Upsilon(j) \text { and } \alpha=\lim _{k \rightarrow \infty} \alpha(k)^{1 / k} .
$$

Then $\alpha$ is called the cogrowth (or cogrowth exponent) of the presentation. By a result of Cohen [1] and Grigorchuk [2] a group $G$ is amenable if and only if $\alpha=2 n-1$. Since 
$1 / \alpha$ is equal to the radius of convergence of the cogrowth series, which is determined by the critical point of the cogrowth series having smallest absolute value, the cogrowth series gives a way to determine whether a group is amenable, although in most cases this is not the most convenient way. The cogrowth and the cogrowth series were studied in $[\mathbf{1}, \mathbf{2}, \mathbf{3}, \mathbf{4}, \mathbf{6}, \mathbf{9}, \mathbf{1 0}]$.

In this paper, we present a way of calculating the cogrowth series of free products of finite and free groups. We explicitly calculate the cogrowth series of the presentations $D_{\infty}=\left\langle x, y \mid x^{2}=y^{2}=i d\right\rangle, \quad P S L_{2}=\left\langle x, y \mid x^{2}=y^{3}=i d\right\rangle$, and $\left(x_{1}, \ldots, x_{n}\left|x_{1}^{k}, \ldots, x_{n}^{k}\right\rangle\right.$ for $k=2,3$ (see Section 3). In some other cases, we use the discriminant to calculate the cogrowth.

The return generating function (see [8]) for a group can be defined in the same way as the cogrowth series if we take the presentation

$$
1 \rightarrow N \rightarrow \mathbf{N}_{n} \rightarrow G \rightarrow 1,
$$

where $\mathbf{N}_{n}$ is the free monoid on $A$. A similar result for those functions was obtained by Gregory Quenell [8].

2. The method. Since the cogrowth series of the presentation of $G$ is the same as the growth series of the set $W=\bigcup_{k \in \mathbf{Z}} W(k)$, we need to find the growth series of $W$. Let $B$ and $C$ be alphabets, and let $x \in C, X \subseteq B^{\star}, Y \subseteq C^{\star}$. Define the operation of substitution of the set $X$ for the letter $x$ in every word of the set $Y$ by

$$
\left.Y\right|_{x=X}=\left\{w_{1} v_{1}^{\varepsilon 1} w_{2} v_{2}^{\varepsilon 2} \ldots w_{n} \mid n \in Z, v_{1}, \ldots, v_{n-1} \in X, w=w_{1} x^{\varepsilon 1} w_{2} x^{\varepsilon 2} \ldots w_{n} \in Y\right\},
$$

where $\varepsilon_{i}= \pm 1$ and $v^{-1}=b_{l}^{-1} \ldots b_{1}^{-1}$ if $v=b_{1} \ldots b_{l}$, where the $b_{i}$ 's are in the alphabet of $X$ (if $x^{-1}$ is present in a word of $Y$ then we assume that for every letter $b$ in the alphabet of $X$, the letter $b^{-1}$ is in the alphabet of $X$ as well). Multiple substitutions are defined by

$$
\left.Y\right|_{\left(x_{1}=X_{1}, \ldots, x_{m}=X_{m}\right)}=\left.\left(\ldots\left(\left.Y\right|_{x_{1}=X_{1}}\right) \mid \ldots\right)\right|_{x_{m}=X_{m}},
$$

where $X_{i} \subseteq B, i=1, \ldots, m$. Let $\mathcal{P}(X)$ denote the power set of $X$.If $w \in\left(A \cup\left\{x_{j}{ }^{ \pm 1}\right\}_{j \in M}\right)^{\star}$, where $M$ is a set of indices, then we will let $I(w) \subseteq M$ denote the set of all indices $i$ such that $x_{i}$ or $x_{i}^{-1}$ is a letter of $w$.

The method we use is as follows.

1. We find a collection of sets $S_{i}, i=0,1,2, \ldots$, where $S_{i} \subseteq S_{i+1} \subseteq A^{\star}$ (for all $i$ ) and partition each $S_{i}$ into substets $S_{i}^{j}, j \in M=\{1, \ldots, m\}$ (where $S_{i}^{j} \subseteq S_{i+1}^{j}$ for all $i$ and $j$ ), so that the following 2 conditions are satisfied.

2. We suppose that we have a function

$$
f: M \rightarrow \mathcal{P}\left(\left(A \bigsqcup\left\{x_{j}^{ \pm 1} \mid j \in M\right\}\right)^{\star}\right),
$$

where $x_{1}, \ldots, x_{m}$ are letters not in $A$, such that

(a) $f$ generates $S_{i+1}$ from $S_{i}$ in the following sense:

$$
\left.f(j)\right|_{\left(x_{1}=S_{i}^{1}, \ldots, x_{m}=S_{i}^{m}\right)}=S_{i+1}^{j} \text {, for all } i \geq 0 \text { and } j \in M \text {. }
$$


(b) $f$ is 1-to- 1 as explained further. Denote $\bigcup_{i=0}^{\infty} S_{i}^{j}$ by $S^{j}$. Then this property can be described as follows: a word in $\left.f(j)\right|_{\left(x_{1}=S^{1}, \ldots, x_{m}=S^{m}\right)}$ can be obtained from only one word $w \in f(j)$ by substituting a word from $S^{j}$ for every $x_{j}$; also, there is only one combination of these words from $S_{j}$ which gives the original word. Formally, the property can be written as follows. Suppose we have words

$$
\begin{aligned}
w & =w_{1} x_{j_{1}} w_{2} x_{j_{2}} \ldots w_{l-1} x_{j_{l-1}} w_{l} \in f(j), \\
w^{\prime} & =w_{1}^{\prime} x_{j_{1}^{\prime}} w_{2}^{\prime} x_{j_{2}^{\prime}} \ldots w_{l^{\prime}-1}^{\prime} x_{j_{l^{\prime}-1}} w_{l}^{\prime} \in f(j),
\end{aligned}
$$

where $w_{i}, w_{i}^{\prime} \in A^{\star}$, and $v_{\alpha} \in \bigcup_{i=0}^{\infty} S_{i}^{j_{\infty}}$ for all $\alpha \in I(w), v_{\alpha}^{\prime} \in \bigcup_{i=0}^{\infty} S_{i}^{j_{\alpha}^{\prime}}$ for all $\alpha \in I\left(w^{\prime}\right)$. Suppose we also have

$$
w_{1} v_{j 1} w_{2} \ldots v_{j_{l}} w_{l}=w_{1}^{\prime} v_{j_{1}^{\prime}}^{\prime} w_{2}^{\prime} \ldots v_{j_{l^{\prime}}^{\prime}}^{\prime} w_{l^{\prime}}^{\prime}
$$

Then

$$
\left(w, w_{1}, v_{j 1}, w_{2}, \ldots, v_{j_{l}}, w_{l}\right)=\left(w^{\prime}, w_{1}^{\prime}, v_{j_{l}^{\prime}}^{\prime}, w_{2}^{\prime}, \ldots, v_{j_{l}^{\prime}}^{\prime}, w_{l}^{\prime}\right)
$$

as ordered sequences. For simplicity, the letter $x$ (with indices) will be reserved for substitution variables, as in the above.

3. We suppose that we can calculate the growth series of $W$ from the growth series of the $S^{j}=\bigcup_{i=0}^{\infty} S_{i}^{j}$.

Then we can calculate the cogrowth series of the presentation using the following result:

THEOREM 1. In the situation described above $f$ induces the following system of functional equations on the series $\gamma_{j}=\gamma\left(S^{j}\right), j \in M$ :

$$
\left\{\begin{array}{ccc}
\gamma_{1} & = & f_{1}^{\star}\left(\gamma_{1}, \ldots, \gamma_{m}\right) \\
\vdots & \vdots & \vdots \\
\gamma_{m} & = & f_{m}^{\star}\left(\gamma_{1}, \ldots, \gamma_{m}\right)
\end{array}\right.
$$

where $f_{j}^{\star}$ is the operator induced by $f(j)$ : if $g_{1}(t), \ldots, g_{m}(t)$ are functions, then

$$
f_{j}^{\star}\left(g_{1}(t), \ldots, g_{m}(t)\right)=\gamma_{\left(x_{1}, \ldots, x_{m}\right)}\left(f(j) ; t, g_{1}(t), \ldots, g_{m}(t)\right) .
$$

Proof. By the generating property 2(a) of $f$ we have:

$$
\left.f(j)\right|_{\left(x_{1}=S_{i}^{1}, \ldots, x_{m}=S_{i}^{m}\right)}=S_{i+1}^{j} \text { for all } j \in M .
$$

Hence,

$$
\left.f(j)\right|_{\left(x_{1}=S^{1}, \ldots, x_{m}=S^{m}\right)}=S^{j} \text { for all } j \in M,
$$

and so, 


$$
\gamma\left(\left.f(j)\right|_{\left(x_{1}=S^{1}, \ldots, x_{m}=S^{m}\right)}\right)=\gamma_{j}
$$

The result follows from the equality

$$
\gamma\left(\left.f(j)\right|_{\left(x_{1}=S^{1}, \ldots, x_{m}=S^{m}\right)}\right)=\gamma_{\left(x_{1}, \ldots, x_{m}\right)}\left(f(j) ; t, \gamma_{1}, \ldots, \gamma_{m}\right) .
$$

The last equality is obtained by repeatedly applying the following result.

Lemma 1. Let $X$ be a set of words in some alphabet containing $x$, let $Y$ be another set of words, and let $Z=\left.X\right|_{x=Y}$. Suppose that if $w=w_{1} x w_{2} x \ldots w_{n 1+1}, w^{\prime}=w_{1}^{\prime}$ $x w_{2}^{\prime} x \ldots w_{n 2+1}^{\prime} \in X$ and $v_{1}, \ldots, v_{n 1}, v_{1}^{\prime}, \ldots, v_{n 2}^{\prime} \in Y$, with

$$
w_{1} v_{1} w_{2} v_{2} \ldots w_{n_{1}+1}=w_{1}^{\prime} v_{1}^{\prime} w_{2}^{\prime} v_{2}^{\prime} \ldots w_{n_{1}+1}^{\prime}
$$

then

$$
\left(w, v_{1}, \ldots, v_{n_{1}}\right)=\left(w^{\prime}, v_{1}^{\prime}, \ldots, v_{n_{2}}^{\prime}\right)
$$

(this is a reformulation of the 1-to-1 property). Then

$$
\gamma(Z ; t)=\gamma_{x}(X ; t, \gamma(Y ; t)) .
$$

Proof. We have:

$$
Z=\left.X\right|_{x=Y}=\left\{\left.w\right|_{x=Y} \mid w \in X\right\}=\left.\bigsqcup_{w \in X}\{w\}\right|_{x=Y} .
$$

The union is disjoint because of the given property of words in $Z$. Hence,

$$
\gamma(Z)=\sum_{w \in X} \gamma\left(\left.\{w\}\right|_{x=Y}\right)
$$

Let $w=b_{1} x b_{2} x \ldots b_{l-1} x b_{l} \in X$, where $b_{i} \neq x$ are in the alphabet of $X$ or empty words. Then

$$
\left.\{w\}\right|_{x=X}=\left\{b_{1} v_{1} b_{2} \ldots v_{l} b_{l} \mid \forall i v_{i} \in Y\right\}=\bigsqcup_{\left(v_{1}, \ldots, v_{l}\right) \in Y \times \ldots \times Y}\left\{b_{1} v_{1} b_{2} \ldots v_{l} b_{l}\right\} .
$$

Again, the union is disjoint because of the given property of words in $Z$. Hence,

$$
\begin{aligned}
\left.\left.\gamma(\{w\})\right|_{x=Y}\right) & =\sum_{\left(v_{1}, \ldots, v_{l}\right) \in Y \times \ldots \times Y} \gamma\left(\left\{b_{1} v_{1} b_{2} \ldots v_{l} b_{l+1}\right\}\right) \\
& =\sum_{v_{1} \in Y} \sum_{v_{2} \in Y} \ldots \sum_{v_{l} \in Y} \gamma\left(\left\{b_{1}\right\}\right) \gamma\left(\left\{v_{1}\right\}\right) \ldots \gamma\left(\left\{v_{l}\right\}\right) \gamma\left(\left\{b_{l+1}\right\}\right) \\
& =\gamma\left(\left\{b_{1} b_{2} \ldots b_{l+1}\right\}\right)\left(\sum_{v \in Y} \gamma(\{v\})\right)^{l} \\
& =\gamma\left(\left\{b_{1} b_{2} \ldots b_{l+1}\right\}\right) \gamma^{l}(Y)=\gamma_{x}\left(\left\{b_{1} x b_{2} \ldots x b_{l+1}\right\} ; t, \gamma(Y)\right) .
\end{aligned}
$$


The last inequality is true since

$$
\gamma_{x}\left(\left\{b_{1} x b_{2} \ldots x b_{l+1}\right\} ; t, x\right)=\gamma\left(\left\{b_{1} b_{2} \ldots b_{l+1}\right\} ; t\right) x^{l} .
$$

Substituting the above expression for $\gamma\left(\left.\{w\}\right|_{x=Y}\right)$ into (2), we obtain

$$
\begin{aligned}
\gamma(Z ; t) & =\sum_{b_{1} x b_{2} \ldots x b_{l} \in X} \gamma\left(\left.\left\{b_{1} x b_{2} \ldots x b_{l}\right\}\right|_{x=Y} ; t\right) \\
& =\sum_{b_{1} x b_{2} \ldots x b_{l} \in X} \gamma_{x}\left(\left\{b_{1} x b_{2} \ldots x b_{l}\right\} ; t, \gamma(Y)\right) \\
& =\gamma_{x}\left(\bigsqcup_{b_{1} x b_{2} \ldots x b_{l} \in X}\left\{b_{1} x b_{2} \ldots x b_{l}\right\} ; t, \gamma(Y)\right) \\
& =\gamma_{x}(X ; t, \gamma(Y)) .
\end{aligned}
$$

This proves the lemma and concludes the proof of Theorem 1 .

REMARK. The following three statements can be useful.

1. The solution of the system of equations (1) can be considered as a fixed point of the operator $F$ :

$$
\left(\begin{array}{c}
g_{1} \\
\vdots \\
g_{m}
\end{array}\right) \rightarrow\left(\begin{array}{c}
f_{1}^{\star}\left(g_{1}, \ldots, g_{m}\right) \\
\vdots \\
f_{m}^{\star}\left(g_{1}, \ldots, g_{m}\right)
\end{array}\right)
$$

2. The system of equations (1) can be considered as iterative: as can be easily seen from the above proof, if we take as a beginning approximation $\gamma_{j}=0$, and substitute it in the right hand side of (1), then we get $\gamma\left(S_{0}\right)$. Substituting in $\gamma\left(S_{0}\right)$ gives $\gamma\left(S_{1}\right)$, and so on.

3. In general a system of functional equations can have more than one solution, but as the previous consideration shows, there exists a unique solution with Maclaurin series having the free coefficient 0 .

In the presentation described in Section $1, a_{i}^{ \pm 1}, i=1, \ldots, n$ are called generators A word in the generators is called a relator if it represents the identity element of $G$ under the map $\psi: A^{\star} \rightarrow G$ induced by the epimorphism $\phi: F_{n} \rightarrow G$. An element of $W$ is called a freely reduced relator. A freely reduced relator is called simple if it is not a concatenation of 2 or more non-trivial $(\neq i d)$ relators.

The following statement is obvious.

Lemma 2. Every relator $v$ is a product of simple relators $v_{1}, \ldots, v_{k}$. Furthermore, the correspondence $v \leftrightarrow\left(v_{1}, \ldots, v_{\mathrm{k}}\right), k \geq 0$ between relators and ordered finite sets of simple relators is a bijection.

\section{Cogrowth series of finite groups.}

Theorem 2. Let $G=\left\{g_{1}, \ldots, g_{N}\right\}$ be a finite group, $g_{1}=i d$, and $P=\left\langle a_{1}, \ldots, a_{n} \mid R\right\rangle$ be a presentation of $G$. Then the relative cogrowth of $P$ with respect to $\vec{a}=\left(a_{1}, \ldots, a_{n}\right)$ is $v_{\vec{a}}(P)=\sum_{k, \varepsilon} \gamma_{g 1 k}^{\varepsilon}+1$, where the $\gamma_{g 1 k}^{\varepsilon}$ are determined by the following system of linear equations in the variables $\gamma_{\beta k}^{\varepsilon}$, where $\beta \in G ; k=1, \ldots, n ; \varepsilon= \pm$ (here $\left.\tilde{\varepsilon}=\varepsilon 1\right)$ 


$$
\left\{\gamma_{\beta k}^{\varepsilon}=\left(\sum_{\substack{k^{\prime} \neq k \\ \varepsilon^{\prime}}} \gamma_{\beta \phi\left(a_{k}\right)^{-\tilde{\varepsilon}}, k^{\prime}}^{\varepsilon^{\prime}}+\gamma_{\beta \phi\left(a_{k}\right)^{-\tilde{\varepsilon}, k}}^{\varepsilon}+\delta_{\beta}^{\phi\left(a_{k}\right)^{\tilde{\varepsilon}}}\right) a_{k}\right\}_{\beta, k, \varepsilon}
$$

In particular the relative cogrowth of $P$ is a rational function.

Proof. In this proof all growth series are assumed to be relative growth series with respect to $\vec{a}$. The result will follow from Theorem 1 . Thus we follow the steps of the method of Section 2.

1. Define $S_{i}$ to be the set of non-empty freely reduced words in $A^{\star}$ (where $A=\left\{a_{1}^{ \pm 1}, \ldots, a_{n}^{ \pm 1}\right\}$ as in Section 1$)$ of length $\leq i+1$. Partition each $S_{i}$ into $S_{i}^{\beta k \varepsilon}$ where $\beta \in G ; 1 \leq k \leq n ; \varepsilon= \pm 1$. Here $S_{i}^{\beta k \varepsilon}$ is defined as the set of words $w \in S_{i}$ such that $\psi(w)=\beta$, and $a_{k}^{\tilde{\varepsilon}}$ (where $\tilde{\varepsilon}=\varepsilon 1$ ) is the last letter of $w$. Obviously, $S_{i}^{\beta k \varepsilon} \subseteq S_{i+1}^{\beta k \varepsilon}$.

2. We define

$$
f(\beta, k, \varepsilon)=\left(\bigcup_{\substack{k^{\prime} \neq k \\ \varepsilon^{\prime}}}\left\{x_{\beta b_{k}^{-\tilde{\varepsilon}, k^{\prime}, \varepsilon^{\prime}}} a_{k}^{\tilde{\varepsilon}}\right\}\right) \bigcup\left\{x_{\beta b_{k}^{-\tilde{\varepsilon}}, k, \varepsilon} a_{k}^{\tilde{\varepsilon}}\right\} \bigcup_{i f \beta=b_{k}^{\tilde{\varepsilon}}}\left\{a_{k}^{\tilde{\varepsilon}}\right\},
$$

where $b_{k}=\phi\left(a_{k}\right)$. Since the right hand side represents the different ways that a word of length $i+1$ which represents $\beta \in G$ can be obtained from a word of length $i$, properties 2(a) and 2(b), required by the method, are satisfied.

3. Since $W=\cup_{k, \varepsilon} \cup_{i=0}^{\infty} S_{i}^{g_{1} k \varepsilon} \cup\{i d\}$, the formula for $v$ follows.

Theorem 1 gives the stated system, where $\gamma_{\beta k}^{\varepsilon}=\gamma_{\vec{a}}\left(S^{\beta k \varepsilon}\right)$ (as in Section 2, $\left.S^{\beta k \varepsilon}=\cup_{i=0}^{\infty} S_{i}^{\beta k \varepsilon}\right)$.

4. The infinite cyclic group in the presentation $\boldsymbol{P}_{\mathbf{0 1}}=\langle\boldsymbol{a}, \boldsymbol{b} \mid \boldsymbol{b}\rangle$. The cogrowth of this presentation has been calculated using a completely different technique in [3]. However, we will need the relative cogrowth with respect to $(a, b)$. Define the height $h(w)$ of a relator $w$ in $P_{01}$ inductively as follows: if $w=w_{1} \ldots w_{k}$, where $w_{i}$ 's are simple relators then $h(w)=\max \left\{h\left(w_{i}\right) \mid i=1, \ldots, k\right\}$; if $w$ is a simple relator then $w=b^{ \pm 1}$ or $w=a^{\varepsilon} w^{\prime} a^{-\varepsilon}$, where $\varepsilon= \pm 1$. In the first case we define $h(w)=1$, and in the second case we define $h(w)=h\left(w^{\prime}\right)+1$. Now we follow the method of Section 2 .

1. Define $S_{i}$ to be the set of simple relators beginning with $a$ of height not more than $i+2$. Further subdivision of $S_{i}$ will not be necessary.

2. In this case, $f$ is a function from a one point set. Identify $f$ with the image of this point. Let $W_{1}$ be the set of non-empty words in $b^{ \pm 1}$ and $x$, in which $x$ doesn't follow itself and $b^{\varepsilon}$ doesn't follow $b^{-\varepsilon}(\varepsilon= \pm 1)$. Define $f$ to be the set of words in $a^{ \pm 1}, b^{ \pm 1}$, and $x$ such that every word $w$ has the form $a w_{1} a^{-1}$, where $w_{1} \in W_{1}$. Then property 2(a) is obvious and 2(b) follows from Lemma 2.

3. By Lemma 2, $W=\left.X\right|_{x=\cup_{i} S_{i}}$, where $X$ is the set of words in $b^{ \pm 1}, x^{ \pm 1}$ in which $x^{\varepsilon}$ doesn't follow itself, and $b^{\varepsilon}$ doesn't follow $b^{-\varepsilon}(\varepsilon= \pm 1)$. It is easy to see that 
$\gamma_{(x, b)}(X)=v_{(x, b)}\left(P_{11}\right)$, where $P_{11}$ is the presentation of the identity group $\langle x, b \mid x, b\rangle$. Hence, using Lemma 1 and the results of Section 3, we have

$$
\gamma_{(x, b)}(X ; t, x, b)=\frac{1+b x-b-x}{1-(3 b x+b+x)}
$$

and

$$
\gamma_{(a, b)}(W ; t, a, b)=\gamma_{(x, b)}\left(X ; t, \gamma_{(a, b)}\left(\cup_{i} S_{i} ; t, a, b\right), b\right)
$$

In the above equations, we used $x, a$, and $b$ as letters of the alphabet in the subscripts of $\gamma$ and as variable names otherwise. Whenever it does not cause confusion, such usage allows faster recognition of the variable that corresponds to a given letter of the alphabet.

Finally

$$
f^{\star}=\gamma_{(a, b, x)}(f)=a^{2} \gamma_{(x, b)}\left(W_{1}\right)
$$

and calculating $\gamma:=\gamma_{(x, b)}\left(W_{1}\right)$ similarly to $\gamma_{(x, b)}\left(P_{11}\right)$, we get the system:

$$
\left\{\begin{array}{c}
\gamma=\gamma_{b}^{+}+\gamma_{b}^{-}+\gamma_{x}^{+}, \\
\gamma_{b}^{+}=\left(\gamma_{x}^{+}+\gamma_{b}^{+}+1\right) b, \\
\gamma_{b}^{-}=\left(\gamma_{x}^{+}+\gamma_{b}^{-}+1\right) b, \\
\gamma_{x}^{+}=\left(\gamma_{b}^{+}+\gamma_{b}^{-}+1\right) x .
\end{array}\right.
$$

Solving this system and applying Theorem 1, we get the following equation on $\gamma:=\gamma_{(a, b)}\left(\cup_{i} S_{i}\right)$ :

$$
\gamma=a^{2} \frac{2 b+3 b \gamma+\gamma}{1-(2 \gamma+1) b}
$$

Solving this equation (using also the fact that $\gamma$ should have Maclaurin series with first coefficient 0 ) and substituting the result in the function obtained at step 3 , after simplifications, we get

$$
\gamma_{(a, b)}\left(P_{01}\right)=\frac{(b+1) \sqrt{1-a^{2}}}{\sqrt{(1+a-b+3 a b)(1-a-b-3 a b)}} .
$$

5. Free product of finite and free groups. Suppose we have a free product of finite and free groups $G$ in the presentation $P=P_{1}{ }^{*} \ldots{ }^{*} P_{n}$, where $P_{j}=\left(a_{j 1}, \ldots, a_{j n_{j}} \mid R_{j}\right)$, $j=1, \ldots, n^{\prime}$; are presentations of finite groups, and $P_{j}=\left\langle a_{j 1}\right\rangle, j=n^{\prime}+1, \ldots, n$ are presentations of free groups on one generator. Also assume that the generators of different $P_{j}$ 's are distinct. Then we can identify any element of $P_{j}$ with its image in $P$ (Prop. 4.1 in [5]). Let $w$ be a simple relator in this presentation. The following procedure defines the 0 'th level of $w$ :

1. Include the first letter of $w$ in the 0 'th level and let $w=b_{1} w_{1}$; where $b_{1}$ is a generator, $w_{1}$ is a word. Let $j$ be such that $b_{1} \in P_{j}$.

2. If we have $w=b_{1} v_{1} b_{2} \ldots v_{l-1} b_{l}(l \geq 1)$, where $b_{1}, \ldots, b_{l}$ are generators included in the 0 'th level, then we are done. 
3. If we have $w=b_{1} v_{1} b_{2} \ldots v_{l-1} b_{l} w_{l}(l \geq 1)$, where $b_{1}, \ldots, b_{l}$ are generators included in the 0'th level, and $w_{l} \neq i d$, then let $v_{l}$ be the shortest initial subword of $w_{l}$ (possibly empty) which is a relator, with the property that the generator $b_{l+1}$ which follows it is in $P_{j}$ (where $j$ has been fixed in the first step). Note, that $v_{l}$ exists since $w$ is a simple relator (see Exercise 1 of 4.1 in [5]). Thus, we have $w=b_{1} v_{1} \ldots v_{1} b_{l+1} w_{l+1}$ for some word $w_{l+1}$ (possibly empty). Include $b_{l+1}$ in the 0 'th level and repeat beginning with step 2 .

This process will stop because the length of each $w_{l}$ is at least 1 less than the length of $w_{l-1}$. Define the 0 'th level of a relator $w$ to be the union of the 0 'th levels of the simple relators of which $w$ is the concatenation.

Now we can state the inductive definition of the level of a relator as follows.

The empty word is a word of level-1. The non-empty relator $w=b_{1} v_{1} \ldots b_{l-1} v_{l-1} b_{l}$, where $b_{1}, \ldots, b_{l}$ are letters that form the 0 'th level is said to be of level $i$ if the maximum level of the $v_{\mathrm{j}}$ 's is $i-1$.

To calculate the cogrowth series of the presentation $P$, we follow the steps of the method of Section 2:

$\underline{\text { Step } 1}$. Define $S_{i}^{j}, i \geq 0, j=1, \ldots, n$ to be the set of non-empty freely reduced relators of level not more than $i$, the 0 'th level of which forms a relator in $P_{j}$. Define $S_{i}=\sqcup_{j=1}^{n} S_{i}^{j}, i \geq 0$. Obviously, $S_{i} \subseteq S_{i+1}$ and $S_{i}^{j} \subseteq S_{i+1}^{j}$ for all $i$ and $j$. Later in the section we will need the following result.

Lemma 3. Any relator $r$ in $P$ can be uniquely written as a concatenation $r_{1} \ldots r_{l}$ of relators satisfying

(i) the 0 'th level of each $r_{i}$ forms a relator in $P_{j}$ for some $j=j(i)$ and

(ii) $j(i) \neq j(i+1)$.

Proof. We have, $r=s_{1} \ldots s_{p}$ for some simple relators $s_{k}$. Let $r_{1}=s_{1} \ldots s_{l}$, where $s_{1}, \ldots, s_{l}$ form a relator in one of the $P_{j}$ 's, and $l$ is such that $s_{l+1} \notin P_{j}$ for that $j$. The result follows by induction on $p$.

Step 2. Define $f(j)$ to be the set of non-empty freely reduced words in $a_{j 1}^{ \pm 1}, \ldots, a_{j n_{j}}^{ \pm 1}, x_{1}, \ldots, x_{j}, \ldots, x_{n}$ such that the following three conditions $(*)$ are satisfied:

(i) neither $x_{i}$ nor $x_{i}^{-1}$ follows itself (for $i=1, \ldots, n$ ),

(ii) if we remove all the $x_{i}$ 's, we get a relator in $P_{j}$; and

(iii) let $w^{\prime}$ be any initial subword having last letter $x_{i}$, and let $w^{\prime \prime}$ be the result of removing all the $x_{i}$ 's from $w^{\prime}$. Then $w^{\prime \prime}$ is not a relator in $P_{j}$.

We claim that the function $f$ defined above is the $f$ referred to in Section 2. We need to cheek the two properties of $f$.

(a) Suppose we have $w=b_{1} w_{l} b_{2} w_{2} \ldots b_{l} \in S_{i+1}^{j}$, where $b_{1}, \ldots, b_{l}$ form the 0 'th level of $w$. Let $j$ be such that $b_{1}, \ldots, b_{l} \in P_{j}$. Then it follows from the procedure of finding the 0 'th level that $w_{k}, k=1, \ldots, l-1$ are products of simple relators the 0 'th level of which is not in $P_{j}$ and of level not more then $i$. Hence,

$$
\left.w \in f(j)\right|_{\left(x_{1}=S_{i}^{1}, \ldots, x_{n}=S_{i}^{n}\right)} .
$$

On the other hand suppose $w$ was obtained from some word 


$$
y=b_{1} y_{1} b_{2} y_{2} \ldots b_{l} \in f(j)
$$

(where $y_{k}$ 's are some words in $x_{k}$ 's) by the substitutions $\left(x_{k}=S_{i}^{k}\right)_{k}$. Then it is of level not more than $i+1$ and $b_{1}, \ldots, b_{l}$ still form the 0 'th level of $w$. Hence $w \in S_{i+1}^{j}$ and the property

$$
\left.f(j)\right|_{x_{1}=S_{i}^{1}, \ldots, x_{n}=S_{i}^{n}}=S_{i+1}^{j}
$$

is proven.

(b) If we have $w_{1} v_{11} \ldots=w_{1}^{\prime} v_{11}^{\prime} \ldots$ as in 2(b) of the method, then the 0 'th level of $w_{1} v_{1} \ldots$ is equal to the 0 'th level of $w^{\prime}{ }_{1} v_{1}^{\prime} \ldots$ This means that

$$
\left(w_{1}, v_{11} \ldots v_{1 n_{1}}, w_{2}, \ldots\right)=\left(w_{1}^{\prime}, v_{11}^{\prime} \ldots v_{1 n_{1}^{\prime}}^{\prime}, w_{2}^{\prime}, \ldots\right) .
$$

Applying Lemma 3 for $v_{k 1} \ldots v_{k n_{k}}=v_{k 1}^{\prime} \ldots v_{k n_{k}^{\prime}}^{\prime}$, we get

$$
\left(v_{k 1}, \ldots, v_{k n_{k}}\right)=\left(v_{k 1}^{\prime}, \ldots, v_{k n_{k}^{\prime}}^{\prime}\right) .
$$

Thus the one to one property is satisfied.

Step 3. By Lemma 3, $W=\left.X\right|_{\left(x_{1}=S^{1}, \ldots, x_{n}=S^{n}\right)}$, where $X$ is the set of words in $x_{1}, \ldots, x_{n}$, such that $x_{i}$ doesn't follow itself ( $X$ includes the empty word). Hence, by Lemma 1, we get

$$
\gamma(W)=\gamma_{\vec{x}}(X)\left(\gamma\left(\cup_{i} S_{i}^{1}\right), \ldots, \gamma\left(\cup_{i} S_{i}^{n}\right)\right)
$$

To calculate $\gamma_{\vec{x}}(X)$, we again use the method of Section 3. By a proof analogous to the one in Section 3, we get that $\gamma_{\vec{x}}(X)=\tilde{\gamma}_{n}$, where $\tilde{\gamma}_{n}$ is determined by the following linear system on $\tilde{\gamma}_{n}, \gamma_{1}, \ldots, \gamma_{n}$ :

$$
\left\{\begin{array}{ccc}
\tilde{\gamma}_{n} & = & \gamma_{x_{1}}+\cdots+\gamma_{x_{n}}, \\
\gamma_{x_{1}}= & \left(\tilde{\gamma}_{n}-\gamma_{x_{1}}+1\right) x_{1}, \\
\vdots & \vdots & \vdots \\
\gamma_{x_{n}} & = & \left(\tilde{\gamma}_{n}-\gamma_{x_{n}}+1\right) x_{n} .
\end{array}\right.
$$

Now, we need to calculate the induced functions. Recall that $f(j)$ consists of freely reduced words in $a_{j 1}^{ \pm 1}, \ldots, a_{j n_{j}}^{ \pm 1}, x_{1}, \ldots, \widehat{x}_{j}, \ldots, x_{n}$ such that conditions $(*)$ are satisfied. Consider words in $f(j)$ in the following way: we have the set of relators of $P_{j}$; then, in each relator of $P_{j}$, we insert words in $x_{1}, \ldots, \widehat{x}_{j}, \ldots, x_{n}$ such that no $x_{i}$ follows itself, so that the relator is freely reduced and conditions (*) are still satisfied. The relative growth of non-empty words which we insert can be calculated as in step 3, and is $\tilde{\gamma}_{n-1}=\tilde{\gamma}_{n-1}\left(x_{1}, \ldots, \widehat{x}_{j}, \ldots, x_{n}\right)$. After that, in the case $1 \leq j \leq n^{\prime}$ we follow the reasoning of Section 3, inducting on the total number of $a_{j i}^{ \pm 1}$ 's instead of length. It is easy to derive the following system of linear equations in the variables $\gamma_{\beta k}^{\varepsilon}$, where $\beta \in G_{j} ; k=1, \ldots, n_{j} ; k^{\prime}=1, \ldots, n_{j} ; \varepsilon, \varepsilon^{\prime}= \pm$ (use $\left.\tilde{\varepsilon}=\varepsilon 1\right)$ : 


$$
\left\{\begin{aligned}
\left\{\gamma_{\beta k}^{\varepsilon}\right. & \left.=\left(\sum_{\frac{k^{\prime} \neq k}{\varepsilon^{\prime}}} \gamma_{\beta a_{j k}^{-\tilde{\varepsilon}}, k^{\prime}}^{\varepsilon^{\prime}}+\gamma_{\beta a_{j k}^{-\tilde{\varepsilon}}, k}^{\varepsilon}\right)\left(\tilde{\gamma}_{n-1}+1\right) a_{j k}+\gamma_{\beta a_{j k}^{-\tilde{\varepsilon}}, k^{\prime}}^{-\varepsilon} \tilde{\gamma}_{n-1} a_{j k}\right\}_{\beta \neq a_{j k}^{\tilde{\varepsilon}}, k, \varepsilon} \\
\gamma_{a_{j k}^{\varepsilon}}^{\varepsilon} & =\left(\sum_{\frac{k^{\prime} \neq k}{\varepsilon^{\prime}}} \gamma_{i d, k^{\prime}}^{\varepsilon^{\prime}}+\gamma_{i d, k}^{\varepsilon}+1\right) a_{j k}
\end{aligned}\right.
$$

with

$$
f_{j}^{\star}\left(x_{1}, \ldots, x_{n}\right)=\gamma_{\vec{x}}(f(j))=\left.\sum_{\varepsilon, k} \gamma_{i d, k}^{\varepsilon}\right|_{\left(a_{j 1}=t, \ldots, a_{j n}=t\right)} .
$$

In the case $n^{\prime}+1 \leq j \leq n$, we use the ideas of Section 4: we look on the relative growth series of the freely reduced words in $a_{j 1}^{ \pm 1}, y$ satisfying the conditions (*), where $y$ stands for words in $x_{i}$ 's. Since there is no $y^{-1}$, and $y$ shall not follow itself the system from Section 4 will be changed to (changing all $a$ 's to $a_{j 1}$ 's and $b$ 's to $y$ 's)

$$
\left\{\begin{aligned}
\gamma & =\gamma_{y}^{+}+\gamma_{x}^{+} \\
\gamma_{y}^{+} & =\left(\gamma_{x}^{+}+1\right) y \\
\gamma_{x}^{+} & =\left(\gamma_{y}^{+}+1\right) x
\end{aligned}\right.
$$

Also, since the level 0 shall be a relator in $P_{j}$,

$$
\gamma_{\left(a_{j 1}, y\right)}(W)=\left.\gamma_{z}(X)\right|_{z=\gamma_{\left(a_{j 1}, y\right)}}\left(\cup_{i} S_{i}\right)
$$

where $X=\left\{z^{k} \mid k \in \mathbf{Z} \backslash\{0\}\right\}$ with $\gamma_{\mathrm{z}}(X)=2 z /(1-z)$. Thus, we finally get

$$
f_{j}^{\star}=\left.\left(\frac{y\left(1-t^{2}\right)-\sqrt{\left(1-t^{2}\right)\left(1-(t+2 t y)^{2}\right)}}{-1+t^{2}+y+3 t^{2} y}-1\right)\right|_{\left(y=\tilde{\gamma}_{n-1}\left(x_{1}, \ldots, \widehat{x}_{j}, \ldots, x_{n}\right)\right)} .
$$

REMARK. The method gives $n$ rational equations; (the equations induced by $f(j)$, $j>n^{\prime}$ can be easily rewritten in rational form as well). However, if we have some $P_{j}=P_{j^{\prime}}$, then the corresponding equations will be the same, so we can eliminate all but one of them. Also, the equation for $P_{j}=\left\langle a_{j 1} \mid a_{j 1}\right\rangle$ is $\gamma_{j}=t$. So, we can eliminate it as well. Furthermore, if one of the presentations of the finite groups is not repeated, we will have an equation $\gamma_{j}=F\left(\gamma_{1}, \ldots, \widehat{\gamma}_{j}, \ldots, \gamma_{n}\right)$, and, we can use it for substitution. Finally, by clearing denominators, we can make the equations polynomial.

\section{Examples.}

ExAmple 1. Let $P_{j}=\left\langle a_{j} \mid a_{j}{ }_{j}\right\rangle$; i.e. we have the presentation $P=$ $\left\langle a_{1}, \ldots, a_{n} \mid a_{1}{ }^{2}, \ldots, a_{n}^{2}\right\rangle$. In this case, all the induced equations are the same, and so we need to solve only one equation with one variable $\gamma=\gamma_{1}=\ldots=\gamma_{n}$.

The induced function will be

$$
f^{\star}(x, \ldots, x)=2 \frac{\left(1+2 \tilde{\gamma}_{n-1}\right) t^{2}}{1-\left(1+2 \tilde{\gamma}_{n-1}\right) t^{2}},
$$


where

$$
\tilde{\gamma}_{n-1}=\tilde{\gamma}_{n-1}(x, \ldots, x)=\frac{(n-1) x}{1-(n-2) x} .
$$

The equation given by Theorem 1 will be (after simplification)

$$
\left(n t^{2}+n-2\right) \gamma^{2}+\left(2 n t^{2}+t^{2}-1\right) \gamma+2 t^{2}=0 .
$$

Solving this equation and choosing the solution with the correct Maclaurin series, we get

$$
\gamma=\frac{1-(2 n+1) t^{2}-\sqrt{1-2(6 n-7) t^{2}+(2 n-1)^{2} t^{4}}}{2\left(n-2+n t^{2}\right)} .
$$

Finally, using the fact that the cogrowth series is $\tilde{\gamma}_{n}\left(\gamma_{1}, \ldots, \gamma_{n}\right)$, we get

$$
v=\frac{(2-n)((2 n-1) t+1)+n \sqrt{1-2(6 n-7) t^{2}+(2 n-1)^{2} t}}{2\left(1-(2 n-1)^{2} t^{2}\right)} .
$$

For the case $n=2$, we have the infinite dihedral group with

$$
v\left(D_{\infty}\right)=\sqrt{\frac{1-t^{2}}{1-9 t^{2}}},
$$

which is amenable. Analysing the singular points of the cogrowth series when $n>2$, we find that the cogrowth of this presentation is

$$
\sqrt{6 n-7-4 \sqrt{(2 n-3)(n-1)}}
$$

and thus, the group is not amenable.

ExAmple 2. Let $P_{j}=\left\langle a_{j} \mid a_{j}{ }^{3}\right\rangle$, and $n>1$; i.e. we have the presentation $P=$ $\left\langle a_{1}, \ldots, a_{n} \mid a_{1}{ }^{3}, \ldots, a_{n}{ }^{3}\right\rangle$. In this case we see that the induced function is

$$
f^{\star}(x, \ldots, x)=\frac{2\left(\tilde{\gamma}_{n-1}+\left(1+2 \tilde{\gamma}_{n-1}\right) t\right) t^{2}}{1-\left(\tilde{\gamma}_{n-1} t+\tilde{\gamma}_{n-1} t^{2}+\left(1+2 \tilde{\gamma}_{n-1}\right) t^{3}\right)}
$$

and all the rest is quite similar to the previous example. We get that the cogrowth series of $P$ is 


$$
v=\frac{-(1+t)\left((n-2)+n t+(n-2)(2 n-1) t^{2}-n \sqrt{R(t)}\right)}{2(1-(2 n-1) t)\left(1+n t+(2 n-1) t^{2}\right)},
$$

where $R(t)=1-2 t+(7-4 n) t^{2}-2(2 n-1) t^{3}+(2 n-1)^{2} t^{4}$

the cogrowth is

$$
\frac{1+2 \sqrt{2 n-2}-\sqrt{4 \sqrt{2 n-2}-3}}{2(2 n-1)}
$$

and thus, the group is not amenable.

ExAmple 3. Let $n=2, P_{1}=\left\langle a \mid a^{2}\right\rangle, P_{2}=\left\langle b \mid b^{3}\right\rangle$; i.e. we have the presentation $P=\left\langle a, b \mid a^{2}, b^{3}\right\rangle$, which is a presentation of the projective special linear group $P S L_{2}(\mathbf{Z})$. In this case the cogrowth series is

$$
v=\frac{(t+1)\left(9 t^{5}-3 t^{4}+8 t^{3}-t^{2}+t-\left(6 t^{2}-t+2\right) \sqrt{R(t)}\right)}{2(3 t-1)\left(3 t^{2}+1\right)\left(3 t^{2}+3 t+1\right)\left(3 t^{2}-t+1\right)},
$$

where $R(t)=81 t^{8}-54 t^{7}+9 t^{6}-18 t^{5}-8 t^{4}-6 t^{3}+t^{2}-2 t+1$.

The cogrowth is $1 / r \approx 2.924984549$, where $r$ is the root of $R(t)$ which is closest to .3418821478 . The group is not amenable.

ExAmple 4. Let again $n=2, P_{1}=\langle a \mid\rangle$ and $P_{2}=\left\langle b \mid b^{2}\right\rangle$; i.e. we have the presentation $P=\left\langle a, b \mid b^{2}\right\rangle$. In this case after simplification, we will have the following equation for the growth series $\gamma$ of freely reduced relators the 0 'th level of which is a word in $a$, $a^{-1}$ :

$$
8 t^{4}+\left(-2+4 t^{2}+30 t^{4}\right) \gamma+\left(-1+33 t^{4}+8 t^{2}\right) \gamma^{2}+2 t^{2}\left(5 t^{2}+3\right) \gamma^{3}=0 .
$$

It is possible to solve this equation in square and cubic roots, but the solution is too long to be written here. Nevertheless, it is relatively simple to find the cogrowth as follows. We know that the cogrowth is $1 / r$, where $r$ is the smallest by absolute value singular point of $\gamma$, since the cogrowth of $P$ is equal to the growth of the freely reduced relators having the 0 'th level consisting of letters $a$ and $a^{-1}$. We know that the singular points of a solution of a polynomial equation are among the roots of the discriminant. Also, we have that the cogrowth of $P$ shall be in the interval $[\sqrt{3}, 3]$, since the cogrowth of a presentation having $2 n$ generators is in the interval $[\sqrt{2 n-1}, 2 n-1]$ (see [1]), and the corresponding singular point will be positive (since we have series with positive coefficients). The above identifies the cogrowth as $1 / r \approx 2.668565567$, where $r$ is the root of

$$
729 t^{12}+2430 t^{10}-945 t^{8}-1052 t^{6}-105 t^{4}+30 t^{2}+1
$$

closest to .3747331572 . This constant also was calculated in [1].

EXAMPLE 5. Let us consider how the cogrowth of the presentations $P_{k}=\left\langle x, y \mid x^{2}, y^{k}\right\rangle$ behaves when $k$ increases. If we calculate the cogrowth of $P_{k}$ as in the Example 4, we will get 


$$
\begin{aligned}
& \alpha_{2}=3.00000000000000, \alpha_{12}=2.67569761213378 \text {, } \\
& \alpha_{3}=2.92498454967618, \alpha_{13}=2.67334760233820 \text {, } \\
& \alpha_{4}=2.84166308599088, \ldots \ldots \ldots \ldots \ldots \\
& \alpha_{5}=2.78382043885166, \alpha_{20}=2.66883920237601 \text {, } \\
& \alpha_{6}=2.74550209084132, \ldots \ldots \ldots \ldots \ldots \\
& \alpha_{7}=2.72015120914404, \alpha_{30}=2.66856981326703 \text {, } \\
& \alpha_{8}=2.70326872061827, \ldots \ldots \ldots \ldots \ldots \ldots \\
& \alpha_{9}=2.69195078417217, \ldots \ldots \ldots \ldots \ldots \ldots \\
& \alpha_{10}=2.68432683301238, \ldots \ldots \ldots \ldots \ldots \\
& \alpha_{11}=2.67917768540835, \ldots \ldots \ldots \ldots \ldots \ldots \\
& \alpha_{12}=2.67569761213378, \alpha_{\infty}=2.66856556650517 \text {, }
\end{aligned}
$$

where $\alpha_{k}$ is the cogrowth of $P_{k}$. As one can see, $\alpha_{k}$ is approaching $\alpha_{\infty}$, when $k \rightarrow \infty$.

AcKnowledgements. The author expresses his deep regard to his research adviser Dr. S. P. Humphries for helpful discussions, checking the results, and assistance in preparation of this article and thanks the referee for useful suggestions.

\section{REFERENCES}

1. J. M. Cohen, Cogrowth and amenability of discrete groups. J. Funct. Anal. 48 (1982), 301-309.

2. R. I. Grigorchuk, Symmetrical random walks on discrete groups, in Multicomponent random systems, ed. R. L. Dobrushin and Ya. G. Sinai (Dekker, New York, 1980), 285-325.

3. S. P. Humphries, Cogrowth of groups and the Dedekind-Frobenius group determinant, Math. Proc. Cambridge Phil. Soc. 121 (1997), 193-217.

4. D. G. Kouksov, On rationality of the cogrowth series, Proc. Amer. Math. Soc., to appear.

5. W. Magnus, A. Karrass and D. Solitar, Combinatorial group theory, 2nd edition (Dover 1976).

6. S. Northshield, Cogrowth of regular graphs, Proc. Amer. Math. Soc. 116, No 1 (1992), 203-205.

7. A. L. T. Paterson, Amenability, Math. Surveys and Monographs, No. 29 (Amer. Math. Soc., 1988). 281.

8. G. Quenell, Combinatorics of free product graphs, Contemp. Math. 173 (1994), 257-

9. R. Szwarc, A short proof of the Gregorchuk-Cohen cogrowth theorem, Proc. Amer. Math. Soc. 106, no 3 (1989), 663-665.

10. W. Woess, Cogrowth of groups and simple random walks, Arch. Math. 41 (1983), $363-370$. 